\title{
ANÁLISE DA QUALIDADE SUPERFICIAL E DIMENSIONAL EM PEÇAS PRODUZIDAS POR MODELAGEM POR DEPOSIÇÃO DE MATERIAL FÚNDIDO
} (FDM)

\section{ANALYSIS OF DIMENSIONAL AND SURFACE QUALITY OF PARTS PROCESSED BY FUSED DEPOSITION MODELING (FDM)}

\author{
Carlos Henrique Pereira Mello* carlos.mello@unifei.edu.br \\ Filipe Natividade Guedes* filipenatividade@yahoo.com.br \\ Vinícius José Mota Noronha* vininoronha88@yahoo.com.br \\ Adriano Akio Kawasaki* adrianokawasaki@yahoo.com.br \\ Thales Eduardo de Sousa Rocha*rocha.eme@gmail.com \\ João Roberto Ferreira* jorofe@unifei.edu.br \\ José Hamilton Chaves Gorgulho Júnior* gorgulho@unifei.edu.br \\ * Universidade Federal de Itajubá
}

\begin{abstract}
Resumo: A prototipagem rápida vem crescendo cada vez mais como uma opção na busca de redução do tempo e dos riscos no processo de desenvolvimento de produtos. Contudo, as peças produzidas por modelagem por deposição de material fundido (FDM) podem sofrer variações na rugosidade e precisão dimensional, dependendo de parâmetros como espessura do filete depositado, sentido da orientação da fabricação da peça, tipo de deposição de camada e geometria do corpo de prova. A literatura é rica em trabalhos dessa natureza a respeito de protótipos rápidos produzidos por outras tecnologias de prototipagem, mas o mesmo não acontece com o FDM. Portanto, a presente pesquisa busca analisar a qualidade superficial e dimensional de protótipos rápidos produzidos com a tecnologia de FDM, por meio de um projeto experimental. De acordo com os dados analisados, foi possível afirmar que algumas variáveis selecionadas como fator principal, ou a interação entre elas, tiveram efeito sobre a resposta rugosidade e nenhum efeito sobre a resposta precisão dimensional. Conclui-se que, na produção de protótipos onde o acabamento superficial for importante, cuidados devem ser tomados na especificação dos parâmetros da máquina, uma vez que a rugosidade da peça final prototipada vai depender fortemente da interação entre os mesmos.
\end{abstract}

Palavras-chave: Prototipagem rápida. Modelagem por deposição de material fundido. FDM. Acabamento superficial. Precisão dimensional.

\begin{abstract}
The rapid prototyping is growing increasingly as an option in the quest to reduce the time and risks in the process of developing products. However, the modeling of parts produced by fused deposition modeling (FDM) can vary in roughness and dimensional accuracy, depending on a few parameters. The literature has plenty of works such as to manifold others rapid prototyping technologies, but the same is not true with the FDM. Therefore, this research seeks to analyze the dimensional and surface quality of parts processed by FDM technology, through an experimental design. According to the data analyzed, it was possible to say that some variables selected as main factor, or the interaction between them, had an effect on the roughness response and no effect on the accuracy dimensional response. It is concluded that to produce prototypes where the surface finish is important, care must be taken in specifying the machine parameters, since the roughness of the parts processed will depend strongly on the interaction between these parameters.
\end{abstract}

Key-words: Rapid prototyping. Fused deposition modeling. FDM. Surface quality. Structural quality. 


\section{INTRODUÇÃO}

O mercado vem obrigando as empresas a lançar novos produtos em cada vez menos tempo. Para atender a esse anseio do mercado, as indústrias podem lançar mão de estratégias para reduzir o tempo de desenvolvimento e uma dessas estratégias pode ser o emprego das tecnologias de prototipagem rápida, reduzindo ainda o risco do desenvolvimento.

Essa tecnologia surgiu em meados da década de 1980, permitindo a obtenção de um protótipo de uma peça ou de um molde, de maneira precisa e relativamente rápida, a partir do modelo tridimensional gerado em um sistema CAD 3D paramétrico.

Tais máquinas, conhecidas como máquinas de prototipagem rápida, permitem obter peças físicas acabadas, de modo automático, de qualquer forma e em dimensões finais, com complexidade e detalhes que não seriam possíveis de se obter em máquinas convencionais de usinagem, ou tornariam sua execução demorada ou complexa em centros de usinagem numericamente comandados.

Dessa forma, tais máquinas possibilitam uma maior velocidade e menor custo na obtenção de protótipos se comparado aos processos tradicionais de usinagem, propiciando uma redução no tempo de desenvolvimento de produtos, além da redução dos riscos inerentes às fases iniciais desse processo.

A prototipagem rápida pode ser aplicada às mais diversas áreas tais como, automotiva, aeronáutica, marketing, restaurações, educação, paleontologia e arquitetura (WOHLERS, 1998), entre outras.

Atualmente existem no mercado diversas tecnologias para prototipagem rápida, desde aquelas que utilizam resinas fotopoliméricas, pós de resinas plásticas, pós metálicos e filamentos em resina plástica. A presente pesquisa se concentra na tecnologia de modelagem por deposição de material fundido (fused deposition modelling - FDM), por ser aquela que oferece equipamentos de menor custo (KOCHAN, 2000), ou seja, ao alcance das pequenas e médias empresas e das instituições de pesquisa.

Contudo, os protótipos rápidos produzidos com a tecnologia FDM podem apresentar variações quanto a sua qualidade estrutural e superficial, dependendo da 
manipulação de parâmetros como a espessura da camada e a posição de construção da peça na plataforma da máquina. Na pesquisa à literatura científica foram encontrados poucos trabalhos (AGARWALA et al., 1996; ARMILLOTTA, 2006; ZIEMIAN; CRAWN III, 2001) a respeito desse tipo de análise para peças fabricadas por prototipagem rápida em FDM.

Visando contribuir com este campo do conhecimento para minimizar esta lacuna na literatura, a presente pesquisa tem por objetivo analisar a qualidade superficial e dimensional de protótipos rápidos produzidos com a tecnologia de modelagem por deposição de material fundido (FDM).

\section{FUNDAMENTAÇÃO TEÓRICA}

\subsection{CONCEITO DE PROTOTIPAGEM RÁPIDA}

A prototipagem rápida $(\mathrm{PR})$ visa produzir protótipos de forma relativamente rápida para inspeção visual, avaliação ergonômica, análise de forma/dimensional e como padrão mestre para a produção de ferramentas para auxiliar na redução de tempo do processo de desenvolvimento de produtos (CHOI; CHAN, 2004). O protótipo de um produto ou componente é parte essencial no seu processo de desenvolvimento, pois possibilita que a análise de sua forma e funcionalidade seja feita em uma fase anterior à produção do ferramental definitivo (VOLPATO et al., 2007).

A PR permite aos projetistas criar rapidamente protótipos concretos a partir de seus projetos, ao invés de figuras bidimensionais, possibilitando um auxílio visual excelente durante a discussão prévia do projeto com colaboradores ou clientes. Além disso, o protótipo pode permitir testes prévios como, por exemplo, ensaios em túnel de vento para componentes aeronáuticos ou análise fotoelástica para se verificar pontos de concentração de tensões na peça.

A utilização da prototipagem rápida no processo desenvolvimento de produtos apresenta, segundo Modeen (2005), os seguintes pontos fortes: sua capacidade de produzir formas tridimensionais complexas e detalhadas; a redução de lead times para peças únicas; e a possibilidade de sua instalação em ambientes não industriais, 
devido a baixa geração de ruídos ou desperdícios. Gondak, Gondak e Zluhan (2005) destacam ainda como pontos fortes: a redução substancial do tempo de lançamento do novo produto no mercado, em razão da diminuição da necessidade de testes e do retrabalho; e a melhoria na manufaturabilidade e a redução de custos no projeto de produtos, pelo fato do protótipo rápido auxiliar a revelar as deficiências e lacunas existentes nos processos de manufatura.

Os processos de prototipagem rápida intensificaram o interesse por protótipos físicos no desenvolvimento de produtos. Chua, Leong e Lim (2003) relatam que na década de 1980 a indústria automobilística necessitava de aproximadamente 16 semanas para completar um projeto de desenvolvimento. Após a introdução das tecnologias de CAD/CAM e CNC este prazo foi reduzido para oito semanas, culminando com uma redução para três semanas, em meados da década de 1990, com a utilização dos sistemas de prototipagem rápida.

Entretanto, Modeen (2005) cita alguns pontos fracos do processo de prototipagem rápida: em tempo real, a velocidade de produção do protótipo é muito lenta. Dependendo do nível de precisão requerido e do tamanho do objeto, o processo pode levar algumas horas ou alguns dias; atualmente, a maioria dos equipamentos somente pode fabricar itens de até $500 \mathrm{~mm}^{3}$ de volume; o número de materiais disponíveis para a prototipagem rápida com adição de materiais é ainda limitado, mas tem aumentado gradativamente; a qualidade final da superfície do protótipo rápido gerado geralmente necessita de um acabamento secundário.

Portanto, o termo "rápido" associado a esses processos é relativo. A construção de alguns protótipos pode levar de três a 72 horas, dependendo do tamanho e complexidade do objeto. Ainda assim esses processos são bem mais rápidos e baratos que os métodos tradicionais, tais como usinagem, que podem requerer um tempo bem maior para fabricar um único protótipo.

Segundo Pham e Gault (1998), as tecnologias de prototipagem rápida se dividem em duas categorias principais: os métodos com remoção de material e com adição de material. A primeira requer um processo no qual uma ferramenta 'subtrai' material, através da utilização de uma variedade de diferentes tipos de fresas, geralmente de um bloco de madeira, resina plástica ou espuma, que é gradualmente reduzido para a réplica física do modelo original desenhado em CAD. No segundo 
caso, o modelo físico é construído sequencialmente, uma camada sobre a outra, até formar uma cópia analógica de seu original digital em CAD.

Atualmente existe um grande número de tecnologias de prototipagem rápida disponível no mercado. Entretanto, sete diferentes tecnologias de prototipagem rápida por adição de material disponíveis comercialmente se destacam, entre elas a Estereolitografia (SLA), a Sinterização Seletiva a Laser (SLS), a Manufatura de Objetos em Lâminas (LOM), a Modelagem por Deposição de Material Fundido (FDM) e a impressão tridimensional (3D Printing) (CHEN, 2000).

Todos os processos de prototipagem rápida por adição de camada atualmente existentes são constituídos por cinco etapas básicas (PALM, 1998), como ilustra a figura 1: criação de um modelo CAD da peça que está sendo projetada; conversão do arquivo CAD em formato STL; fatiamento do arquivo STL em finas camadas transversais; construção física do modelo, empilhando-se uma camada sobre a outra; limpeza e acabamento do protótipo.

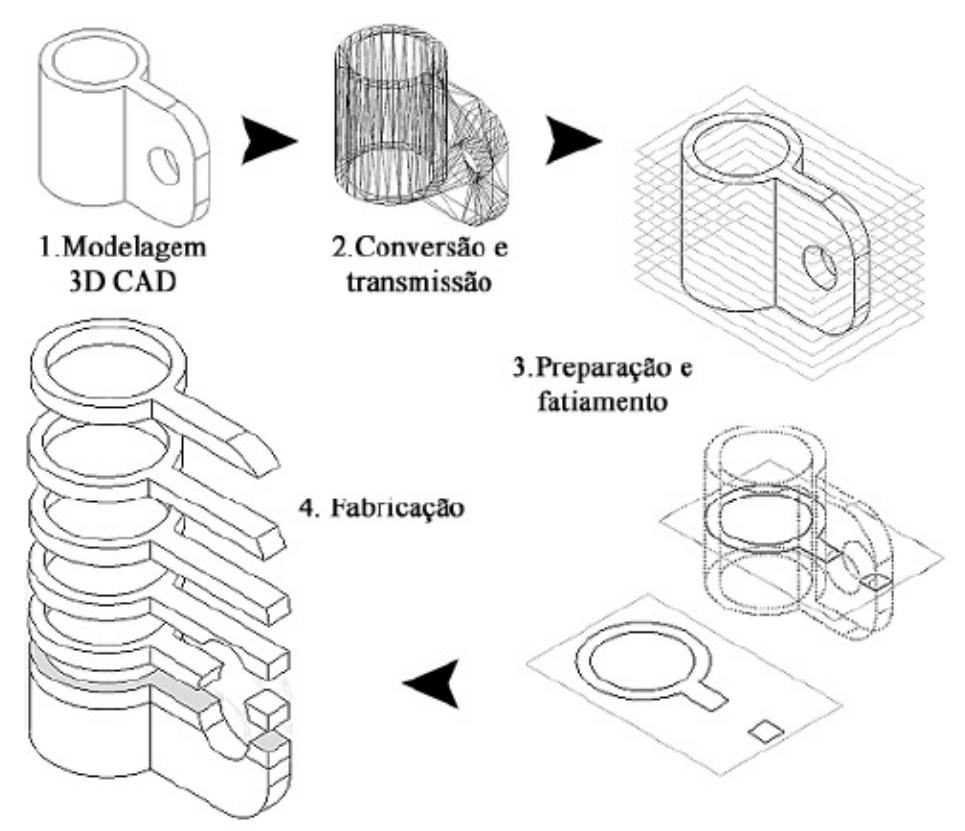

Figura 1: Ciclo básico do processo de PR por adição de camadas Fonte: Beal (2002)

A presente pesquisa se concentra na tecnologia de modelagem por deposição de material fundido (fused deposition modelling - FDM), por ser aquela 
que oferece equipamentos de menor custo (KOCHAN, 2000), ou seja, ao alcance das pequenas e médias empresas e das instituições de pesquisa.

\subsection{MODELAGEM POR DEPOSIÇÃO DE MATERIAL FUNDIDO (FDM)}

A FDM se baseia na deposição, sobre uma plataforma, de camadas resultantes do aquecimento e amolecimento de filamentos do material plástico destinado à confecção do modelo. Simultaneamente, outros fios amolecidos vão formando suportes para as superfícies livremente suspensas do modelo, a fim de que elas possam ser construídas. Os arames destinados ao modelo são de ABS, elastômeros ou cera, enquanto os destinados aos suportes são uma mistura de ABS e cal.

A máquina para a FDM possui uma plataforma, revestida de uma espuma densa e flexível, que se movimenta no sentido vertical (eixo Z) e um cabeçote provido de dois bicos extrusores de arames aquecidos: um para alimentar as camadas do modelo e outro para a construção automática dos suportes. Esses arames ficam estocados dentro da máquina, em ambiente a vácuo aquecido, pois a umidade do material dentro do bico extrusor poderia causar formação de bolhas, que impediria a continuidade de sua deposição pelo bico. Os bicos extrusores, que na verdade, funcionam como uma resistência, são alimentados por esses filamentos através de duas guias giratórias ligadas a um motor, as quais vão transferindo para eles os arames estocados no rolo.

O software da FDM é um misto CAD/CAM e não é integrado à máquina. Esta é conectada ao computador, com o sistema CAM, que monitora constantemente os comandos de construção. Para cada camada geram-se coordenadas ou caminhos pelos quais o bico extrusor vai depositando os fios fundidos. Ao final de cada camada a plataforma desce e o cabeçote inicia a deposição de mais material para a outra camada, repetindo a operação até a conclusão do modelo (ARTIS, 2006). As resinas termoplásticas adequadas a esse processo incluem poliéster, polipropileno, ABS, elastômeros e cera usada no processo de fundição por cera perdida (GORNI, 
2003). A figura 2 ilustra o processo de modelagem por deposição de material fundido.

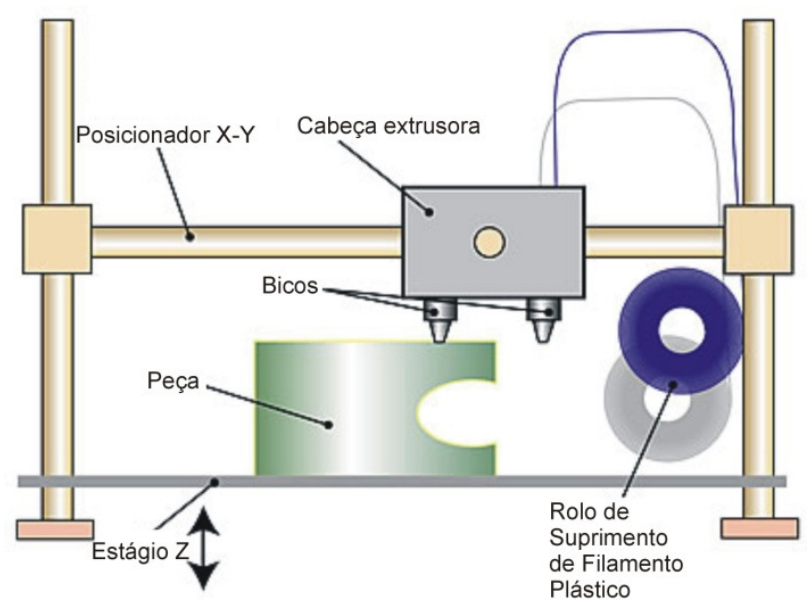

Figura 2: O processo de modelagem por deposição de material fundido Fonte: Artis (2006)

O processo não desperdiça material durante ou após a produção do protótipo, exigindo pouca necessidade de limpeza e permitindo sua instalação em ambientes não industriais (WOHLERS, 1993). Ao longo do processo de deposição foi observado por Armillota (2006) que este depende de alguns fatores, como:

- características das máquinas, a precisão da máquina pode ser obtido com a capacidade da mesma de começar e parar, de acordo com o perfil da peça;

- a taxa de deposição não pode ser menor do que os limites de acordo com as dimensões tais como o diâmetro da ponta;

- o encolhimento e as tensões internas da peça é proveniente do resfriamento e da solidificação;

- a face onde se encontra o suporte possui uma irregularidade ao ponto de não poder ser medida com o rugosímetro.

A literatura indica que durante 0 processo de prototipagem podem surgir alguns fenômenos, principalmente na FDM, que trata-se de deposição de material 
com temperatura elevada, ao ponto do material ficar com uma textura pastosa, e seu posterior resfriamento.

Um fenômeno comumente observado é o efeito escada (staircase), que implica na descontinuidade da superfície da peça prototipada, devido ao efeito do seu resfriamento e da deposição de camadas (WENBIN, TSUI; HAIQING, 2005). Esse efeito ocorre entre duas camadas adjacentes (layers) como ilustra a figura 3.

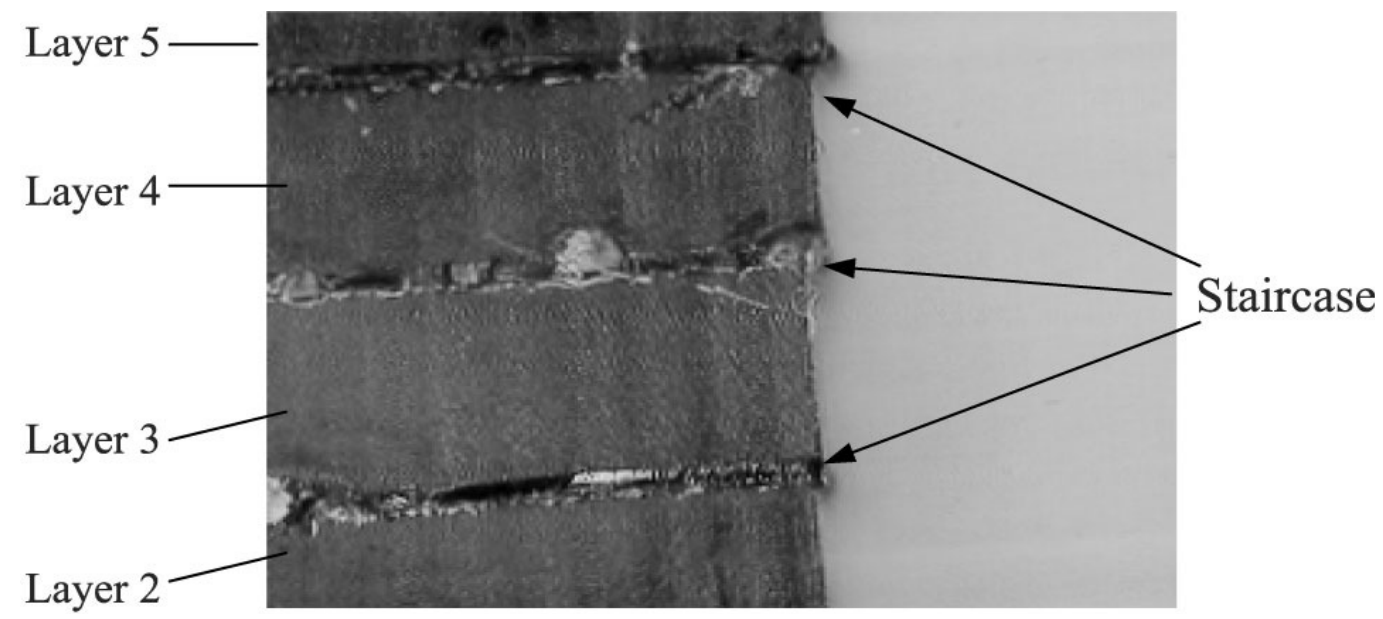

Figura 3: Efeito escada (staircase)

Fonte: Wenbin, Yong e Haiqing (2005)

Ziemian e Crawn III (2001) realizaram um experimento que visou à observação do processo FDM e os efeitos das diferentes variáveis do processo, através de um experimento fracional fatorial. Foram observadas quatro variáveis, sendo elas a orientação da construção, a espessura da camada, a largura da camada e a estratégia de preenchimento do interior, sendo que para cada uma delas foram estabelecidos três níveis correspondentes a uma classificação alta, média e baixa. A máquina utilizada em tal experimento foi a FDM 2000, fabricada pela Stratasys, e o material foi o ABS P400. Foram investigadas diferentes formas de corpos de prova como, por exemplo, retangular, cilíndrico, prismático, correspondendo a três diferentes volumes de modo a representar faces planas que estão perpendiculares ou não entre si (retângulos e prismas) e também superfícies arredondadas (cilindros).

Os autores daquele trabalho analisaram as influências das variáveis no tempo de construção, precisão dimensional e qualidade da superfície. 
Quanto aos resultados obtidos em relação à precisão dimensional, foi observado que não há forte relação entre a resposta e as variáveis selecionadas. A análise estatística realizada se mostrou substancialmente melhor para as partes retangulares e cilíndricas e o efeito mais significante foi determinado como sendo a estratégia de preenchimento do interior.

Investigações adicionais indicam que a precisão da FDM2000 pode estar significativamente mais relacionada a fatores não incluídos no estudo realizado. No entanto, as evidências sugerem que a localização do corpo de prova dentro da mesa $X Y$ da máquina tem um impacto na torção e na distorção do material durante a fabricação. Ziemian e Crawn III (2001) acreditam que isso é relevante devido aos parâmetros de resfriamento do material e do fluxo de ar dentro do envelope de trabalho.

Segundo Kechagias (2007), a tecnologia de prototipagem rápida conhecida por Manufatura de Objetos em Lâminas (Laminated Object Manufacturing - LOM) possui impacto direto (na espessura da camada, na temperatura, na plataforma, na velocidade de calor e na velocidade do laser) ou indireto (na velocidade de alimentação e na velocidade da plataforma) durante o processo de deposição de camadas na superfície vertical.

Apesar de ser um processo diferente, pode-se associar analogamente que a temperatura, a velocidade de calor e a plataforma pode interferir no processo de modelagem por deposição de material fundido (FDM). No quadro 1 e na tabela 1 Kechagias (2007) mostra a interferência de cada característica de acordo com a rugosidade (parâmetro Ra).

Tabela 1 - Resultados encontrados na pesquisa de Kechagias (2007)

\begin{tabular}{|c|c|c|c|c|c|c|c|c|}
\hline No & LT & HT & PR & HS & LS & FS & PS & $\operatorname{Ra}(\mu \mathrm{m})$ \\
\hline 1 & 0.1 & 170 & 0.3 & 70 & 150 & 50 & 25 & 6.4 \\
\hline 2 & 0.1 & 170 & 0.3 & 140 & 180 & 100 & 50 & 13.9 \\
\hline 3 & 0.1 & 190 & 0.4 & 70 & 150 & 100 & 50 & 11.4 \\
\hline 4 & 0.1 & 190 & 0.4 & 140 & 180 & 50 & 25 & 15.5 \\
\hline 5 & 0.2 & 170 & 0.4 & 70 & 180 & 50 & 50 & 12.9 \\
\hline 6 & 0.2 & 170 & 0.4 & 140 & 150 & 100 & 25 & 11.1 \\
\hline 7 & 0.2 & 190 & 0.3 & 70 & 180 & 100 & 25 & 20.6 \\
\hline 8 & 0.2 & 190 & 0.3 & 140 & 150 & 50 & 50 & 19.2 \\
\hline \multicolumn{8}{|c|}{ Mean } & 13.9 \\
\hline
\end{tabular}

Fonte: Kechagias (2007) 


\begin{tabular}{l|l|l}
\hline Símbolo & Significado & Unidade \\
\hline LT & Espessura da camada (layer thickness) & $\mathrm{Mm}$ \\
\hline HT & Temperatura do cabeçote (heater temperature) & ${ }^{\circ} \mathrm{C}$ \\
\hline PR & Retração da plataforma (platform retract) & $\mathrm{mm}$ \\
\hline HS & Velocidade do cabeçote (heater speed) & $\mathrm{mm} / \mathrm{seg}$ \\
\hline LS & Velocidade do laser (laser speed) & $\mathrm{mm} / \mathrm{seg}$ \\
\hline FS & Velocidade do alimentador (feed speed) & $\mathrm{mm} / \mathrm{seg}$ \\
\hline PS & Velocidade da plataforma (platform speed) & $\mathrm{mm} / \mathrm{seg}$ \\
\hline Ra & Rugosidade média (roughness average) & $\mu \mathrm{m}$ \\
\hline
\end{tabular}

Quadro 1: Nomenclatura dos símbolos utilizados na tabela 1

Fonte: Kechagias (2007)

Utiliza-se o parâmetro rugosidade média ( $R a$ - Roughness average), principalmente quando é necessário o controle contínuo da rugosidade e em superfícies em que $o$ acabamento apresenta sulcos de usinagem bem orientados.

A utilização deste parâmetro possui como principais vantagens ser o parâmetro mais utilizado em todo o mundo, sua aplicação quase que universal e que para a maioria das superfícies o valor da rugosidade nesse parâmetro está de acordo com a curva de Gauss, que caracteriza a distribuição de amplitude (LIMA, 2005).

O parâmetro rugosidade média $(R z)$ possui algumas vantagens na sua utilização. Ele informa, por exemplo, a distribuição média da superfície vertical, em perfis periódicos, define muito bem a superfície e ainda faz com que riscos isolados sejam considerados apenas parcialmente, de acordo com o número de pontos isolados (LIMA, 2005).

$\mathrm{Na}$ presente pesquisa foram utilizados apenas os parâmetros de espessura de camada e rugosidade média para posterior comparação com os resultados obtidos por (2007).

\section{MÉTODO DE PESQUISA ADOTADO}

O método de pesquisa adotado para a presente pesquisa foi o experimental fracional fatorial. 
O procedimento experimental adotado visou analisar algumas propriedades dimensionais e físicas de peças fabricadas pelo processo de prototipagem rápida por FDM.

Inicialmente, foram construídos corpos de prova pelo processo de Modelagem por Deposição de Material Fundido (FDM) na máquina de prototipagem rápida produzida pela Stratasys, modelo SST 768 , situada no Laboratório de Produto e Prototipagem Rápida da Universidade Federal de Itajubá (UNIFEI). Os corpos de prova foram numerados e os testes e ensaios foram realizados de forma aleatória para obter resultados mais confiáveis, sendo que, dessa forma, minimizou-se o risco de haver previsão dos mesmos.

Após uma análise de normas específicas e na literatura científica, foram escolhidas as dimensões e as geometrias a serem utilizadas nos corpos de provas. As figuras 4 e 5 mostram o formato dos corpos de prova utilizados. Essa escolha se baseou nos processos de medição a serem futuramente realizados.

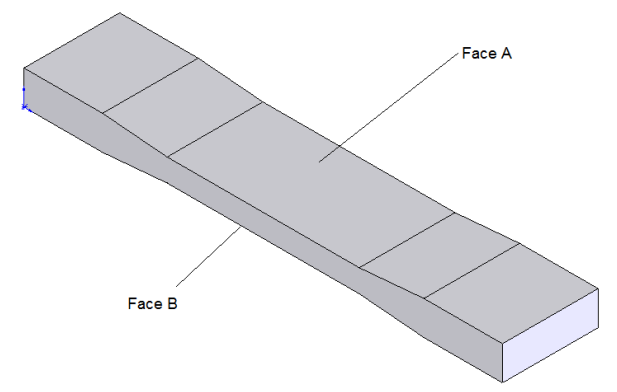

Figura 4: Corpo de prova I

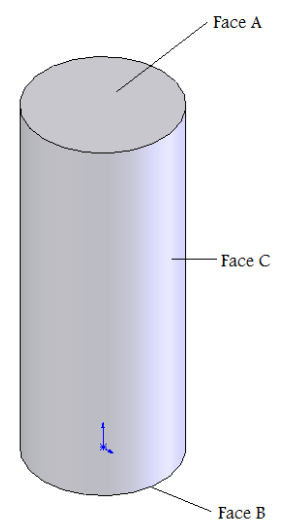

Figura 5: Corpo de prova T 
Os parâmetros considerados como fatores foram:

- espessura do filete depositado pela máquina de FDM (0,25 ou 0,33 mm);

- peça aerada ou normal/sólida;

- posição de construção, sendo a $0^{\circ}$ ou $90^{\circ}$;

- geometria do corpo de prova achatada (I) ou circular (T).

Na tabela 2, gerada pelo Minitab 14, é possível verificar a variação dos parâmetros que foram utilizados, na ordem em que as peças foram produzidas.

Tabela 2 - Parâmetros do experimento

\begin{tabular}{|c|c|c|c|}
\hline Espessura & Camada & Ângulo & Perfil \\
\hline 0,33 & Normal & $90^{\circ}$ & 1 \\
\hline 0,33 & Normal & $90^{\circ}$ & T \\
\hline 0,25 & Normal & $90^{\circ}$ & T \\
\hline 0,33 & Normal & $90^{\circ}$ & T \\
\hline 0,33 & Aerada & $90^{\circ}$ & I \\
\hline 0,33 & Aerada & $90^{\circ}$ & $\mathrm{T}$ \\
\hline 0,25 & Aerada & $90^{\circ}$ & $\mathrm{T}$ \\
\hline 0,33 & Aerada & $0^{\circ}$ & $\mathrm{T}$ \\
\hline 0,33 & Aerada & $0^{\circ}$ & T \\
\hline 0,25 & Normal & $90^{\circ}$ & $\mathrm{T}$ \\
\hline 0,25 & Normal & $0^{\circ}$ & T \\
\hline 0,25 & Aerada & $90^{\circ}$ & I \\
\hline 0,25 & Normal & $0^{\circ}$ & T \\
\hline 0,33 & Normal & $0^{\circ}$ & $\mathrm{T}$ \\
\hline 0,25 & Aerada & $90^{\circ}$ & T \\
\hline 0,33 & Aerada & $90^{\circ}$ & I \\
\hline 0,33 & Aerada & $90^{\circ}$ & T \\
\hline 0,33 & Aerada & $0^{\circ}$ & I \\
\hline 0,25 & Aerada & 0 은 & T \\
\hline 0,25 & Aerada & 0 은 & $\mathrm{T}$ \\
\hline 0,33 & Normal & $0^{\circ}$ & I \\
\hline 0,33 & Normal & $0^{\circ}$ & T \\
\hline 0,25 & Normal & $90^{\circ}$ & I \\
\hline 0,25 & Aerada & $0^{\circ}$ & I \\
\hline 0,25 & Normal & $90^{\circ}$ & I \\
\hline 0,25 & Normal & $0^{\circ}$ & I \\
\hline 0,25 & Aerada & $0^{\circ}$ & I \\
\hline 0,25 & Aerada & $90^{\circ}$ & I \\
\hline 0,33 & Normal & $0^{\circ}$ & I \\
\hline 0,33 & Aerada & $0^{\circ}$ & I \\
\hline 0,33 & Normal & $90^{\circ}$ & I \\
\hline 0,25 & Normal & 0 & I \\
\hline
\end{tabular}

Revista Produção Online, v.10, n.3, p. 504-523, set., 2010 
Por meio do programa Minitab 14 foi gerada uma listagem dos 32 corpos de prova organizados de forma aleatória para a realização dos experimentos.

No processo experimental da presente pesquisa foi analisada a rugosidade dos diferentes corpos de prova por meio de medições realizadas no Laboratório de Metrologia Dimensional da UNIFEI, pelo parâmetro RzDin, utilizando-se o rugosímetro da marca Taylor Hobson, modelo Surtronic 3+. As propriedades dimensionais dos diferentes corpos de prova foram ensaiadas utilizando-se a Máquina de Medir por Coordenadas da marca Mitutoyo, modelo B-251.

Posteriormente, foram feitas análises estatísticas, para verificar o efeito de cada variação dos parâmetros dos corpos de prova ensaiados. Os corpos de prova foram desenhados no programa SolidWorks, versão 2006, com as dimensões de acordo com o estipulado no projeto e, em seguida, os mesmos inseridos no programa CatalistEX da máquina de prototipagem rápida para serem produzidos em ABS.

Os corpos de prova com espessura de camada de $0,25 \mathrm{~mm}$ e de $0,33 \mathrm{~mm}$ tiveram de ser produzidos separadamente, uma vez que a máquina não permite que peças com espessuras de camadas diferentes sejam produzidas de uma só vez.

\section{ANÁLISE DOS RESULTADOS}

Através dos dados coletados pôde-se fazer as análises estatísticas de influência através dos parâmetros considerados:

- Fator $A$ corresponde à espessura do filete extrudado $(0,25 \mathrm{~mm}$ ou $0,33 \mathrm{~mm})$;

- Fator B corresponde ao tipo de deposição da camada (sólida ou aerada);

- Fator $\mathrm{C}$ corresponde ao ângulo em que o corpo de prova foi prototipado $\left(0^{\circ}\right.$ ou $90^{\circ}$ );

- Fator D corresponde ao perfil do corpo de prova (achatado ou cilíndrico).

Esses parâmetros foram analisados individualmente como fator principal e interações de segunda ordem da seguinte forma: 
- A: variando a espessura do filete;

- B: variando o tipo de deposição da camada;

- C: variando o ângulo de prototipagem;

- D: variando o perfil do corpo de prova;

- AB: considerando a interação entre a espessura do filete e o tipo de deposição da camada;

- AC: considerando a interação entre a espessura do filete e o ângulo de prototipagem;

- AD: considerando a interação entre a espessura do filete e o perfil do corpo de prova;

- BC: considerando a interação entre o tipo de deposição da camada e o ângulo de prototipagem;

- BD: considerando a interação entre o tipo de deposição da camada e o perfil do corpo de prova;

- CD: considerando a interação entre o ângulo de prototipagem e o perfil do corpo de prova.

Foi utilizado um $p$-value de 0,10 , uma vez que na maior parte das pesquisas realizadas, o $p$-value utilizado é de $5 \%$ ou $10 \%$.

\subsection{Análise dos Parâmetros Dimensionais}

Foram gerados três gráficos referentes ao comprimento de todos os 32 corpos de prova, à espessura dos 16 corpos de prova achatados e ao diâmetro dos 16 corpos de prova cilíndricos.

Analisando-se os resultados dos efeitos padronizados no comprimento e na espessura dos corpos de prova, percebe-se que nenhuma das variáveis selecionadas, consideradas como fator principal, ou das respectivas interações, teve efeito sobre a resposta da precisão dimensional, já que nenhum dos fatores e/ou interação demonstrou afetar significativamente a resposta. 


\subsection{Análise dos Parâmetros de Rugosidade}

Foram plotados seis tipos de gráficos, sendo três com o parâmetro Ra e outros três com o parâmetro RzDin.

\subsubsection{Face $A$}

As variáveis selecionadas como fator principal, tipo da deposição da camada (sólida ou aerada) e o ângulo em que o corpo de prova foi prototipado ( $0^{\circ}$ ou $90^{\circ}$ ), ou a interação entre elas, teve efeito sobre a resposta rugosidade $(\mathrm{Ra})$, pois ultrapassou a linha de significância de 1,721 . Os demais fatores principais ou suas interações não demonstraram afetar significativamente a resposta.

Os efeitos padronizados na face $A$ dos corpos de prova, percebe-se que nenhuma das variáveis selecionadas, consideradas como fator principal ou das respectivas interações, teve efeito sobre a resposta da rugosidade $(R z)$, já que nenhum dos fatores e/ou interação demonstrou no gráfico de Pareto afetar significativamente a resposta (a linha de significância de 1,721). A figura 6 ilustra as análises na fase $A$ dos corpos de prova.

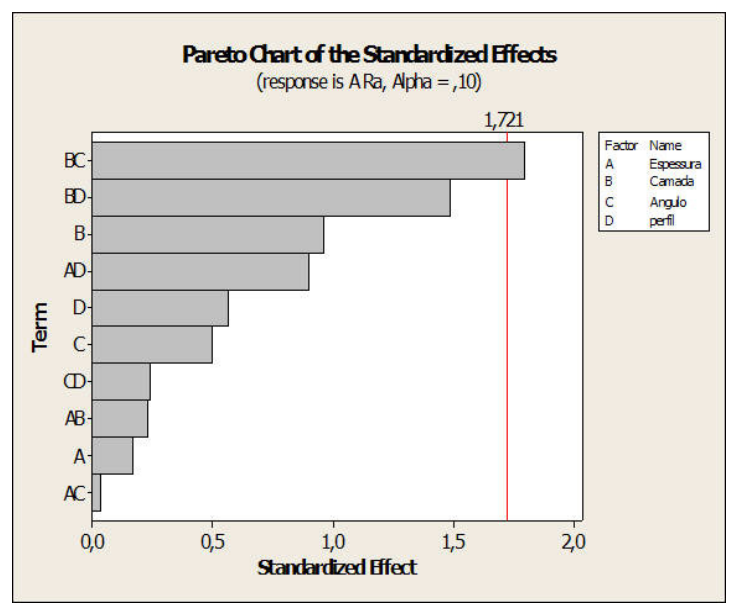

Influência em Ra

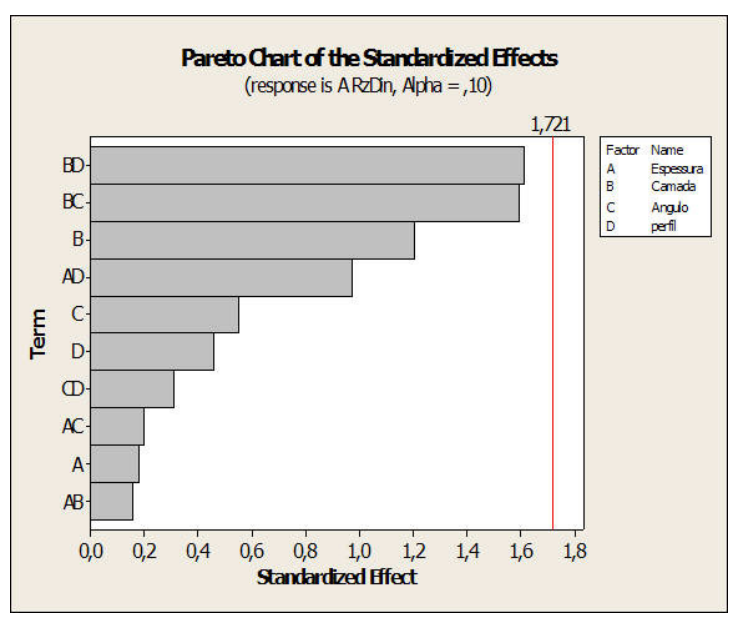

Influência em $R z$

Figura 6: Gráfico de Pareto da influência em Ra e Rz na face A 


\subsubsection{Face B}

A figura 7 mostra que as variáveis selecionadas como fator principal, espessura do filete extrudado $(0,25$ ou $0,33 \mathrm{~mm})$ e o tipo de deposição da camada (sólida ou aerada), ou a interação entre elas, teve efeito sobre a resposta rugosidade Ra e $R z$, respectivamente, pois ambas ultrapassaram a linha de significância de 1,721. Os demais fatores principais ou suas interações não demonstraram afetar significativamente a resposta.

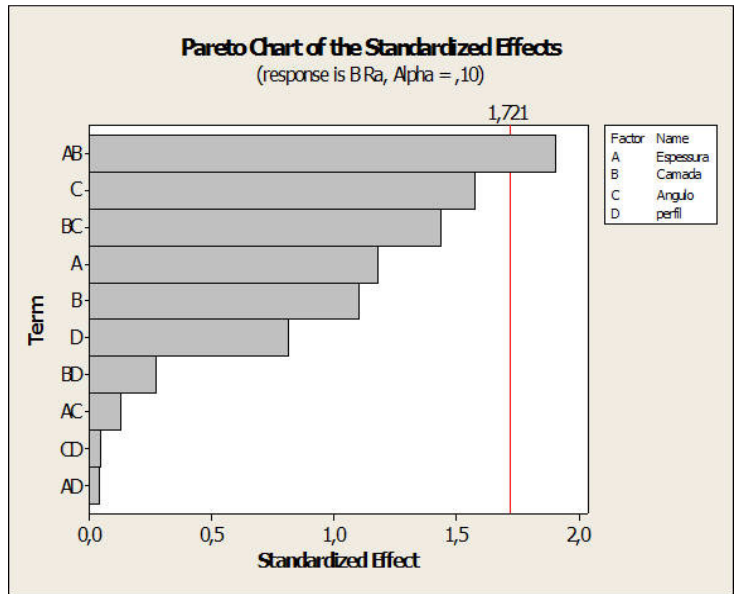

Influência em Ra

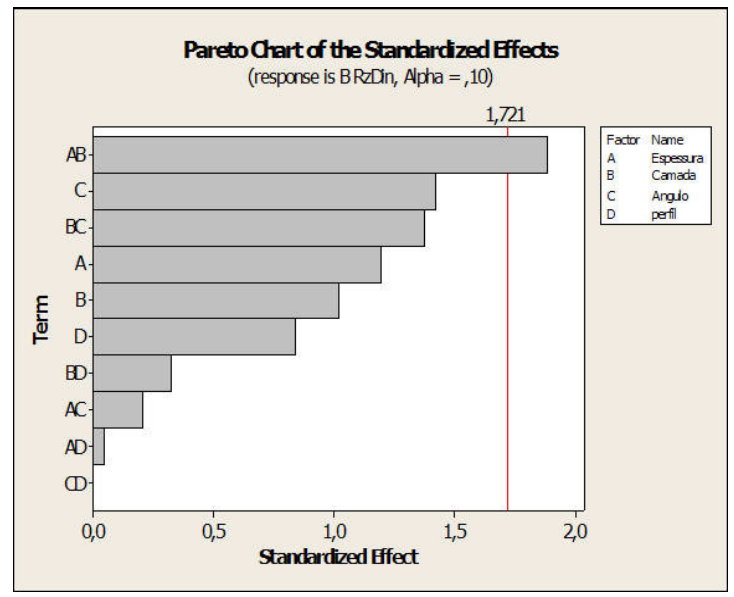

Influência em Rz

Figura 7: Gráfico de Pareto da influência em Ra e Rz na face B

\subsubsection{Face C}

Constatou-se que nenhuma das variáveis selecionadas, consideradas como fator principal ou suas respectivas interações, teve efeito sobre a resposta da rugosidade $\mathrm{Ra}$ e $\mathrm{Rz}$ na face $\mathrm{C}$.

\section{CONCLUSÕES}

Em relação a precisão dimensional, pôde-se concluir que os fatores selecionados em seus efeitos principais ou nas interações realizadas não afetam essa resposta. Isso confirma outros estudos similares já realizados, como os de Ziemian e Crawn III (2001), porém utilizando uma máquina FDM 2000, ao contrário 
deste trabalho que utilizou uma máquina Dimension SST 768, ambas fabricadas pela Stratasys. Em ambos os estudos a matéria prima empregada foi o filamento ABS P400.

Portanto, o presente estudo sugere que a precisão dimensional não é afetada por nenhum dos parâmetros considerados para a máquina Dimension SST 768. Uma limitação dessa pesquisa foi não avaliar a influência da eventual contração das peças produzidas, uma vez que após o resfriamento da câmara (envelope) onde as peças são produzidas, a temperatura sofre um decréscimo de $70{ }^{\circ} \mathrm{C}$ (temperatura de trabalho) até a temperatura ambiente (quando as peças são retiradas da máquina). Entretanto, essa eventual contração pareceu não influenciar nos resultados obtidos na presente pesquisa.

Algumas das peças foram ainda submetidas a um processo de retirada de material de suporte, realizada em uma cuba de ultra-som, em um banho salino $\mathrm{pH}$ próximo de 13), a uma temperatura de $58 \stackrel{\circ}{\circ}$, por aproximadamente quatro horas. Os resultados sugerem que tal procedimento também não interfere na precisão dimensional final das peças prototipadas.

Em relação a resposta rugosidade, o fenômeno do efeito escada (staircase) foi observado nos corpos de prova prototipados, pois se trata de uma forma de deposição com altas temperaturas e resfriamentos dos protótipos, assim havendo um movimento relativo entre as camadas por não possuírem a mesma taxa de resfriamento, indo de encontro ao que Wenbin, Tsui e Haiqing (2005) já haviam observado.

É importante ressaltar que na face em que o protótipo se encontrava junto com o suporte não pôde ser feita a medição de rugosidade, pois a mesma encontrava-se em condições tão ruins que, em muitos casos, não foi considerado como uma rugosidade. Isso foi comprovado quando não foi possível fazer a medição das peças no rugosímetro. Esse fenômeno coincide com os resultados encontrados no estudo similar realizado por Armillota (2006).

De acordo com os dados analisados, é possível afirmar que algumas variáveis selecionadas como fator principal, ou a interação entre elas, tiveram efeito sobre a resposta rugosidade ( $\mathrm{Ra}$ ou $\mathrm{Rz}$ ). O fator tipo da deposição de camada 
(sólida ou aerada) mostrou-se significativo tanto na face $A$ quanto na face $B$, quando interagindo com outros fatores, influenciando na resposta (rugosidade).

Portanto, na produção de protótipos em que a qualidade do acabamento superficial for importante, cuidados devem ser tomados na especificação dos parâmetros da máquina a serem definidos na preparação da mesma, em especial, o tipo de deposição de camada e a posição de construção do protótipo. Este trabalho mostrou que a deposição de camada sólida, ao invés da aerada, melhora a rugosidade da peça nas faces $\mathrm{A}$ e $\mathrm{B}$, uma vez que neste tipo de construção as camadas são mais densas, ao contrário da deposição no formato aerado.

Para futuras pesquisas, sugerem-se ensaios destrutivos como a tração, compressão e impacto para se avaliar a participação dos fatores estudados nesta pesquisa nessas respostas.

\section{AGRADECIMENTOS}

Os autores agradecem a Fapemig (processos EDT-538/07 e TEC-PPM00043-08) e ao CNPq pelos recursos fornecidos na forma de projetos de pesquisa financiados e bolsas de iniciação científica, sem as quais a realização desta pesquisa não seria possível.

\section{REFERÊNCIAS}

AGARWALA, M. K.; JALALABAD, V. R.; LANGRANA, N. A.; SAFARI, A.; WHALEN, P. J.; DANFORTH, S. C. Structural quality of parts processed by fused deposition. Rapid Prototyping Journal, v. 2, n. 4, p. 4-19, 1996.

ARMILLOTTA, A. Assessment of surface quality on textured FDM prototypes. Rapid Prototyping Journal, v. 12, n. 1, p. 35-41, 2006.

ARTIS. Tecnologias de prototipagem: estereolitografia SLA. Brasília: Clínica de Odontologia Integrada Artis, 2006. Disponível em: <http://www.artis.com.br>. Acesso em: 25 de maio de 2008.

BEAL, V. E. Avaliação do uso de insertos obtidos por estereolitografia na moldagem de pós metálicos por injeção. Dissertação (Mestrado) - Programa de 
Pós-graduação em Engenharia Mecânica, Departamento de Engenharia Mecânica, UFSC, Florianópolis, 2002, 87p.

CHEN, L. C. Reverse engineering in the design of turbine blades - a case study in applying the MAMDP. Robotics and Computer Integrated Manufacturing, v. 16, n. 2-3, p. 161-167, 2000.

CHOI, S. H.; CHAN, A. M. M. A virtual prototyping system for rapid product development. Computer-Aided Design, n. 36, p. 401-412, 2004.

GONDAK, M. O.; GONDAK, R. O.; ZLUHAN, G. P. Otimização do desenvolvimento de produto através da Prototipagem Rápida na obtenção de ferramental e protótipos em curto espaço de tempo. CONGRESSO BRASILEIRO DE GESTÃO DE DESENVOLVIMENTO DE PRODUTO, 5., 2005. Anais...Curitiba, 2005.

GORNI, A. A. Introdução à Prototipagem Rápida e seus Processos. Revista Plástico Industrial, p. 230-239, mar., 2003.

KECHAGIAS, F. An experimental investigation of the surface roughness of parts produced by LOM process. Rapid Prototyping Journal, v.13, n. 1, p.17-22, 2007.

LIMA, V. T. Apostila prática de metrologia. Universidade do Rio Verde Graduação em Engenharia Mecânica, 2005. Disponível em: $<$ http://www.engmec.fesurv.br/ Metrologia2005 (Pr\%E1tica).pdf >. Acesso em: 11 abr. 2009.

MODEEN, T. CADCAMing. The use of rapid prototyping for the conceptualization and fabrication of architecture. Automation in Construction, n. 14, p. 215-224, 2005.

PALM, W. Rapid prototyping primer: the learning factory. 1998. Disponível em: <http:// www.mne.psu.edu/lamancusa/rapidpro/primer/chapter2.htm> . Acesso em: 17 maio 2008.

PHAM, D. T.; GAULT, R. S. A comparision of rapid prototyping technologies. International Journal of Machine Tools and Manufacture, n. 38, p. 1257-1287, 1998.

VOLPATO, N. et al. Prototipagem rápida: tecnologias e aplicações. São Paulo: Edgard Blücher, 2007.

WENBIN, H.; TSUI, L. Y.; HAIQING, G. A study of the staircase effect induced by material shrinkage in rapid prototyping. Rapid Prototyping Journal, v. 11, n. 2, p. 82-89, 2005.

WOHLERS, Terry T. Rapid prototyping systems. EUROPEAN RAPID PROTOTYPING CONVENTION, 1., 1993. Proceedings . Paris, França, 1993. 
Disponível em: <http://www.wohlersassociates.com/overview.html>. Acesso em 19 maio 2008.

WOHLERS, T. Rapid prototyping \& tooling: worldwide progress report. Colorado, USA, 1998.

ZIEMIAN, C. W.; CRAWN III, P. M. Computer aided decision support for fused deposition modeling. Rapid Prototyping Journal, v. 7, n. 3, p. 138-147, 2001.

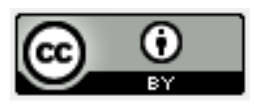

Artigo recebido em 11/04/2009 e aceito para publicação em 15/07/2010. 